$\mathrm{DE}$

M E D I C I N A

T R O P I C A L

$\mathrm{DE}$

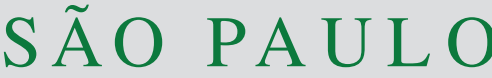

JOURNAL OF THE SÃO PAULO INSTITUTE OF TROPICAL MEDICINE

'Universidade de São Paulo, Faculdade de Odontologia, Departamento de Estomatologia, São Paulo, São Paulo, Brazil

¿Universidade de São Paulo, Instituto de Medicina Tropical de São Paulo, Laboratório de Micologia Médica (LIM 53), São Paulo, São Paulo, Brazil

${ }^{3}$ Universidade de São Paulo, Faculdade de Medicina, Departamento e Instituto de Psiquiatria, São Paulo, São Paulo, Brazil

Correspondence to: Wladimir Gushiken de Campos

Universidade de São Paulo, Faculdade de Odontologia, Departamento de Estomatologia, Av. Professor Lineu Prestes, 2227, CEP 05508-001, São Paulo, SP, Brazil

Tel: +55 $113091-7883$

E-mail: wladimir.campos@usp.br

Received: 28 November 2019

Accepted: 27 April 2020

\section{Oral yeast colonization in patients with eating disorders: commensal acquisition or due to purgative habits?}

Camilla Vieira Esteves ${ }^{(1)}$, Roseli Santos de Freitas ${ }^{2}$, Wladimir Gushiken de Campos ${ }^{\circledR 1}$, Natali Shimabukuro', Danilo Yamamoto Thomaz ${ }^{\circledR}$, Taki Cordas ${ }^{3}$, Gil Benard ${ }^{2}$, Andrea Lusvarghi Witzel ${ }^{1}$, Celso Augusto Lemos ${ }^{1}$

\section{ABSTRACT}

Oral problems are common in patients diagnosed with Eating Disorders (ED) and still require better elucidation. We aimed to analyze the prevalence of oral Candida spp in individuals with ED. The sample of the study was comprised of 30 women with purgative habits and 15 without purgative habits. Samples of the oral cavity were collected by sterile cotton swab rubbed on soft tissues and teeth. Yeasts were isolated on Sabouraud dextrose agar. Yeasts were isolated from the oral cavity of $53 \%$ of the patients yielding 75 yeast isolates; of these, 43 were identified by conventional mycological methods: C. parapsilosis $(\mathrm{n}=19)$, C. glabrata $(\mathrm{n}=16)$, Rhodotorula $\mathrm{sp}(\mathrm{n}=6)$, . famata $(\mathrm{n}=2)$. The remaining 32 isolates were presumptively identified as $C$. albicans or $C$. dubliniensis and required mass spectrometry for the final differentiation: 28 isolates were confirmed as $C$. albicans and four as $C$. dubliniensis. Among the control group, only four subjects (26.7\%) were found to harbor C. albicans. The four $C$. dubliniensis isolates were from two patients, one that was only colonized and the other, with severe ED, was diagnosed with an oral candidiasis as demonstrated by the presence of pseudohyphae on the direct mycological exam from different sites. The increased rate of isolation of non-albicans species, such as C. glabrata, C. parapsilosis, and C. dubliniensis in the oral cavity from ED patients with nutritional deficiency may suggest that purgative habits of these patients can lead to changes in normal flora and predispose to oral candidiasis.

KEYWORDS: Eating disorders. Oral candidiasis. Candida. Infection.

\section{INTRODUCTION}

Eating Disorders (ED) are biologically based on serious mental illnesses and are associated with high medical and psychiatric comorbidity and increased mortality ${ }^{1}$. The major eating disorders are Anorexia Nervosa (AN) and Bulimia Nervosa $(\mathrm{BN})^{1}$, AN having the highest mortality rate of all mental disorders. AN is divided into two subgroups: I. Purgative AN: Induction of vomit, and II. Restrictive AN. The criteria to diagnose AN in a person comprise persistent restriction of energy intake leading to significantly low body weight (in context of what is minimally expected for age, sex, developmental trajectory and physical health) or an intense fear of gaining weight or of becoming fat, or persistent behavior that interferes with weight gain (despite having significantly low weight). BN, on the other hand, is an eating disorder in which a person engages in uncontrollable episodes of overeating, followed by behaviors to prevent weight gain ${ }^{2,3}$.

ED comprise a wide group of clinical conditions that lead to alterations in the mouth, salivary glands, as well as perioral tissues ${ }^{4}$. Consequently, oral disorders 
such as dental erosion, saliva acidification due to the gastric juice's low $\mathrm{pH}$, and alteration of the normal flora are not infrequent ${ }^{2,3,5}$.

Humans are colonized by a diversity of commensal microbes, comprising the human microbiome ${ }^{5}$. The knowledge on the composition, function, and range of variation across multiple body sites has begun to assemble a rich picture of commensal host-microbe and microbemicrobe interactions as well as their roles in human health and disease and their potential as diagnostic and therapeutic tools. The microbiota of the oral cavity can cause varying degrees of oral infections in individuals with risk factors, and several clinically important Candida species that are part of the oral microbiota can undergo uncontrolled proliferation depending on the diet, smoking habits, antibacterial therapy and immunocompromising conditions ${ }^{6}$. C. albicans is the most prevalent fungal species in the oral cavity ${ }^{7}$, and an opportunistic pathogen: depending on the host's immunity it can cause oral mucositis and trush ${ }^{5}$. Candida spp. have most commonly been found on the dorsal surface of the tongue of the oral cavity, followed by palatal and oral mucosa. Petrovic et al. ${ }^{8}$ verified that the subgingival areas are potential reservoirs of different Candida spp in type 2 diabetes patients and healthy subjects.

This study analyzed the prevalence of Candida spp and described the fungi colonization from the oral cavity of non-HIV infected women with ED as compared with women without ED.

\section{PATIENTS AND METHODS}

Thirty women attending the Eating Disorders Clinic (EDC) were enrolled in the study from August (2014) to June (2015). The diagnostic criteria for Anorexia Nervosa and Bulimia Nervosa (DSM-5) ${ }^{1}$ were used. The age ranged from 22 to 45 years (mean $\pm \mathrm{SE}, 32 \pm 1.7$ ). Exclusion criteria were as follows: diabetes mellitus, pregnancy, total denture users, HIV-seropositivity or any other immunosuppressive comorbidity or medication, and current antifungal and antibacterial treatments. To homogenize the study population, we recruited only women with purging habits. Part of this cohort was previously evaluated regarding specific oral and dental alterations ${ }^{9}$. The control group consisted of 15 clinically healthy women without oral mucosal lesions and eating disorders, with- the same age range of the patients. These women were recruited from the Oral Medicine Dental Clinic (CDO-FOUSP) in the same period of time, as the patients. The age ranged from 20 to 43 years $(30.7 \pm 2.6)$. The study was approved by the Institutional Ethics Committee ( $\mathrm{N}^{\circ}$ 919.061/2014).

\section{Oral collection and mycological diagnosis}

Oral samples were collected from the dorsum of the tongue, palate, buccal mucosae, and supragingival area separately by means of sterilized swabs (one swab per location). For the control group, samples were collected from mucosae and dorsum of the tongue, also separately. After collection, samples were transferred to individual tubes containing $5 \mathrm{~mL}$ of sterile saline $(0.9 \% \mathrm{NaCl})$ and transported to the Laboratory of Medical Investigation (LIM-53), School of Medicine, University of Sao Paulo, where they were processed. Due to the numerous collection sites from each patient, each collected sample was inoculated first on Sabouraud dextrose agar (SDA) tubes supplemented with cloramphenicol Difco ${ }^{\text {TM }}$ (Maryland, USA). A fragment of each positive culture was then transferred to CHROMagar Candida medium plates (CHROMagar, Paris, France), and carefully distributed by streaking to obtain pure colonies and facilitate presumptive identification of the species. Whenever a plate exhibited growth of colonies with different colors, each colony was further analyzed separately. In parallel, a smear of the oral material was Gram-stained for direct examination (DME). Oral colonization by Candida spp was considered when there were only yeast forms, whereas oral infection was considered when, in addition to yeast forms, there were pseudohyphae or a large number of yeast forms with buds. Isolates were identified by traditional mycological methods, based on the atlas of Clinical Fungi ${ }^{10}$ (Micromorphology on Cornmeal Agar and Biochemical tests).

Carbon source assimilation was assessed by the commercial kit API 20 C AUX (Biomérux SA, Marcy-I'Etoile, France). The isolates were maintained at the fungal collection from the Medical Mycology Laboratory (LIM 53). C. albicans ATCC 90028 was used as control in all the identification tests.

\section{Differentiation between C. albicans and C. dubliniensis}

As $C$. albicans and $C$. dubliniensis develop green color colonies in chromogenic medium ${ }^{10}$, complementary methodologies are necessary to discriminate these species. The thermotolerance test at $45^{\circ} \mathrm{C}^{11,12}$ is used to indicate the presence of $C$. albicans which, unlike $C$. dubliniensis, can grow in SDA at $45^{\circ} \mathrm{C}$. The assimilation tests (xylose and threalose $)^{11}$ help differentiate both species because $C$. albicans can assimilate these sugars, whereas C. dubliniensis does not. Finally, the tolerance test ${ }^{13}$ is based on the growth in hypertonic medium, which inhibits the growth of $C$. dubliniensis, but not of $C$ albicans. 
Micromorphology was analyzed on Staib Agar media containing Guizotia abyssinica seeds ${ }^{10}$, because C. dubliniensis has the capacity to produce abundant spherical chlamydospores on chains or catenulate, whereas C. albicans colonies do not produce chlamydospores.

Mass spectrometry was carried out as a confirmatory methodology for the identification of isolates suggestive of C. dubliniensis.

\section{Mass spectrometry}

Mass spectrometry (MS) analysis was performed with Candida isolates previously cultivated on Sabouraud dextrose agar (BD, Franklin Lakes, NJ, USA) at $30{ }^{\circ} \mathrm{C}$ for $24 \mathrm{~h}$. The protein extraction was carried out according to a standard protocol with some modifications. One to three yeast colonies were harvested with a $10 \mu \mathrm{L}$ loop and suspended in $300 \mu \mathrm{L}$ of ultrapure water plus $900 \mu \mathrm{L}$ of absolute ethanol (Merck, Darmstadt, Germany). After centrifugation at $17.949 \mathrm{~g}$ (Eppendorf 5804R, SigmaAldrich Chemie, Munich, Germany) for $3 \mathrm{~min}$, the pellet was completely dried by vacuum, centrifuged at $45{ }^{\circ} \mathrm{C}$ and re-suspended in $50 \mu \mathrm{L}$ of formic acid (70\%) (Sigma, St. Louis, MO, USA). After vortex homogeneization $50 \mu \mathrm{L}$ of acetonitrile (Sigma) were added, and the suspension was centrifuged at $17.949 \mathrm{~g}$ (Eppendorf 5804R, SigmaAldrich Chemie, Munich, Germany) for $3 \mathrm{~min}$ and $1.2 \mu \mathrm{L}$ of clear supernatants were spotted in quadruplicate onto the MALDI target plate. After air-drying, each sample was overlaid with $1.2 \mu \mathrm{L}$ of matrix solution (saturated solution of $\alpha$-cyano-4-hydroxy cinnamic acid in organic solvent [50\% acetonitrile and 2.5\% trifluoroacetic acid], Sigma) and air-dried completely before MS analysis. Calibration was performed before each experiment using a Bruker bacterial test standard (Bruker Daltonics GmbH). Mass spectra were generated with the microflex MALDI-TOF mass spectrometer (Bruker Daltonics, Bremen, Germany), and were compared to the main spectra of Candida species available in the Biotyper v3.1 database (Bruker Daltonics). Mass spectrometry results were expressed in log-score values, which are considered acceptable for genus identification between 1,700 and 1,999, and for species identification at values $>2,000$.

\section{RESULTS}

According to the current diagnostic criteria of AN and $\mathrm{BN}^{1}$, seven patients had anorexia and 23 patients had bulimia. Hospitalization due to ED complications was required in five patients. The decayed, missing, and filled teeth (DMF) index mean was 7.1 ${ }^{9}$. In the control group, all the 15 patients presented good oral hygiene, with mean DMF of 2.4.

Yeasts were isolated from the oral cavity in 53\% $(16 / 30)$ of the patients. There were 75 yeast isolates; of these, 43 were identified by conventional mycological methods such as micromorphology on Cornmeal Agar and biochemical tests: $C$. glabrata $(\mathrm{n}=16), C$. parapsilosis $(\mathrm{n}=19)$, Rhodotorula $\mathrm{sp},(\mathrm{n}=6)$, C. famata $(\mathrm{n}=2)($ Table 1). Interestingly, we observed that from the 16 C. glabrata isolates, in five of them this species was associated with simultaneous isolation of other Candida species (C. glabrata/C. albicans, $\mathrm{n}=2$, C. glabrata/C. parapsilosis, $\mathrm{n}=2$, and C. glabrata/C. dubliniensis, $\mathrm{n}=1)($ Table 1). C. parapsilosis, in turn, was associated with C. albicans $(\mathrm{n}=5)$, and Rhodotorula $\mathrm{sp}(\mathrm{n}=6)$. The remaining 32 isolates yielded green colonies presumptive of $C$. albicans or C. dubliniensis and the conventional mycological methods were not able to discriminate between them. Of note, DME from only one patient (\#14ED) revealed the presence of pseudohyphae from different sites (tongue, supragingival area, and oral mucosa), suggestive of infection. This patient received antifungal treatment for oral candidiasis. On the other hand, all DME from the control group were negative; Candida spp were isolated on Sabouraud agar in 4 out of 15 subjects in this group (26.7\%). All four isolates were identified as $C$. albicans and grew in both, the buccal mucosae and dorsum of tongue.

Table 1 - Presence of different fungal species of medically important yeast in the oral cavity from 30 patients with eating disorders. Oral mucosa referred to the content of the tongue, palate and oral mucosa.

\begin{tabular}{lcc}
\hline \multirow{2}{*}{ Microbial growth (Species) } & \multicolumn{2}{c}{ Specimen (N) } \\
\cline { 2 - 3 } C. albicans & 21 & 0 \\
C. glabrata & 10 & 1 \\
C. famata & 2 & 0 \\
C. dubliniensis & 2 & 1 \\
C. parapsilosis & 5 & 1 \\
C. parapsilosis + C. albicans & $5 / 5$ & 0 \\
C. parapsilosis + Rhodotorula sp & $6 / 6$ & 0 \\
C. glabrata + C. parapsilosis & $2 / 2$ & 0 \\
C. glabrata + C. albicans & $2 / 2$ & 0 \\
C. dubliniensis + C. glabrata & 0 & $1 / 1$ \\
\hline Total & 70 & 5 \\
\hline
\end{tabular}

In the remaining 32 isolates, further differentiation between $C$. albicans and $C$. dubliniensis was attempted by analysis of the colony pigmentation on CHROMagar 
Candida medium associated with micromorphology on Cornmeal Agar. Twenty isolates were suggestive of C. dubliniensis due to the presence of green or dark green colonies and pseudomycelium with several chlamydospores mainly in the terminal part of the chains or catenulate on Cornmeal Agar, while the other 12 were identified as C. albicans due to the light green colony and visualization of only one terminal chlamydospore. The twenty presumptive C. dubliniensis isolates were subsequently plated on STAIB Agar for further identification of the species. Only four of these isolates (from patients: \#2ED and \#14ED) displayed pseudomycelia with one to five chlamydospores, mostly in the terminal part of the chains, and wrinkled cultures, strongly suggestive of $C$. dubliniensis. The remaining sixteen did not display chlamydospores, arguing against their identification as C. dubliniensis. Further attempts to confirm the identification were done by using the absence of growth in hypertonic ASD medium $(6.5 \% \mathrm{NaCl})^{12}$ and the thermotolerance at $45{ }^{\circ} \mathrm{C}$, as recommended ${ }^{10,11}$. However, these tests were also inconclusive (Table 2 and Figure 1). Therefore, we submitted these twenty isolates to mass spectrometry (microflex MALDI-TOF mass spectrometer). The four isolates were definitively identified as $C$. dubliniensis, while the other sixteen were identified as C. albicans. (Table 2 and Figure 1)

\section{DISCUSSION}

Over the course of our lives, humans are colonized by a tremendous diversity of commensal microbes, which profoundly affect human health and disease. The rich commensal host-microbe and microbe-microbe interactions, as well as, their roles in human health and disease and their potential as diagnostic and therapeutic tools are in continuous investigation ${ }^{14}$.

Nutritional deficiency such as low levels of iron, zinc, vitamin $\mathrm{K}$, and the purging habits observed in patients with ED can cause alterations in the commensal oral microflora ${ }^{14}$.

The transition from commensalism to pathogenicity depends on the selective expression of different virulence factor, such as the hemolytic activity ${ }^{15}$, besides alterations in the natural microbial habitat associated with host's commorbidities 5 .

Isolation of yeasts from the oral mucosal from our ED patients was positive in $53 \%$ of them. C. albicans (40\%), followed by C. glabrata (22.9\%) and C. parapsilosis (20\%) were the most frequent species. This agrees with previous studies which showed an increasing general incidence of non-albicans species in recent years ${ }^{16-18}$. Another study ${ }^{19}$ found positive carriage of Candida species in $74.6 \%$ of patients with eating disorders; however, the purgative

Table 2 - Phenotypic characteristics and mass spectrometry analysis of $C$. dubliniensis isolates in the study.

\begin{tabular}{|c|c|c|c|c|c|c|c|}
\hline Isolate & Oral location & $\begin{array}{c}\text { Colony } \\
\text { color on } \\
\text { CHROMagar }\end{array}$ & $\begin{array}{l}\text { Growth at } \\
45^{\circ} \mathrm{C}\end{array}$ & $\begin{array}{l}\text { ASD medium } \\
6.5 \% \mathrm{NaCl}\end{array}$ & $\begin{array}{l}\text { Chlamydospore } \\
\text { Cornmeal Agar/ } \\
\text { STAIB Agar }\end{array}$ & Xyl Tre & $\begin{array}{l}\text { MALDI-TOF } \\
\text { Score Value }\end{array}$ \\
\hline $\begin{array}{c}01 \text { ED } \\
\text { C. albicans }\end{array}$ & $\begin{array}{c}\text { Tongue and } \\
\text { supragingival area }\end{array}$ & Dark green & $+/-$ & + & $2+1-$ & + & 2.156 \\
\hline $\begin{array}{c}02 \mathrm{ED} \\
\text { C. dubliniensis }\end{array}$ & $\begin{array}{c}\text { Tongue and } \\
\text { supragingival area }\end{array}$ & Dark green & $+/-$ & - & $4+/ 4+$ & + & 2.073 \\
\hline $\begin{array}{l}13 \text { ED } \\
\text { C. albicans }\end{array}$ & $\begin{array}{c}\text { Tongue and } \\
\text { supragingival area }\end{array}$ & Green & + & + & $2+/-$ & + & 2.280 \\
\hline $\begin{array}{c}14 \mathrm{ED} \\
\text { C. dubliniensis }\end{array}$ & $\begin{array}{c}\text { Tongue, supragingival } \\
\text { area and buccal } \\
\text { mucosa }\end{array}$ & Green & - & - & $3+/ 2+$ & + & 2.174 \\
\hline $\begin{array}{c}15 \text { ED } \\
\text { C. albicans }\end{array}$ & $\begin{array}{c}\text { Tongue, supragingival } \\
\text { area, palate and buccal } \\
\text { mucosa }\end{array}$ & Green & + & + & $2+/-$ & + & 2.187 \\
\hline $\begin{array}{c}16 \text { ED } \\
\text { C. albicans }\end{array}$ & $\begin{array}{c}\text { Tongue, supragingival } \\
\text { area, palate and buccal } \\
\text { mucosa }\end{array}$ & Green & + & + & $2+/-$ & + & 2.280 \\
\hline $\begin{array}{c}\text { C. albicans ATCC } \\
90028\end{array}$ & & Green & + & + & $2+/-$ & + & 2.207 \\
\hline
\end{tabular}

+ = positive; - = Negative; $\mathbf{2 +}-\mathbf{4 +}=$ Number of chlamydospores; $\mathbf{X y l}=$ Acid production from $x y l o s e ;$ Tre = Acid production from trehalose. Score Value: 1,700 and 1,999 = acceptable for genus identification; $>2,000=$ genus and species identification. 


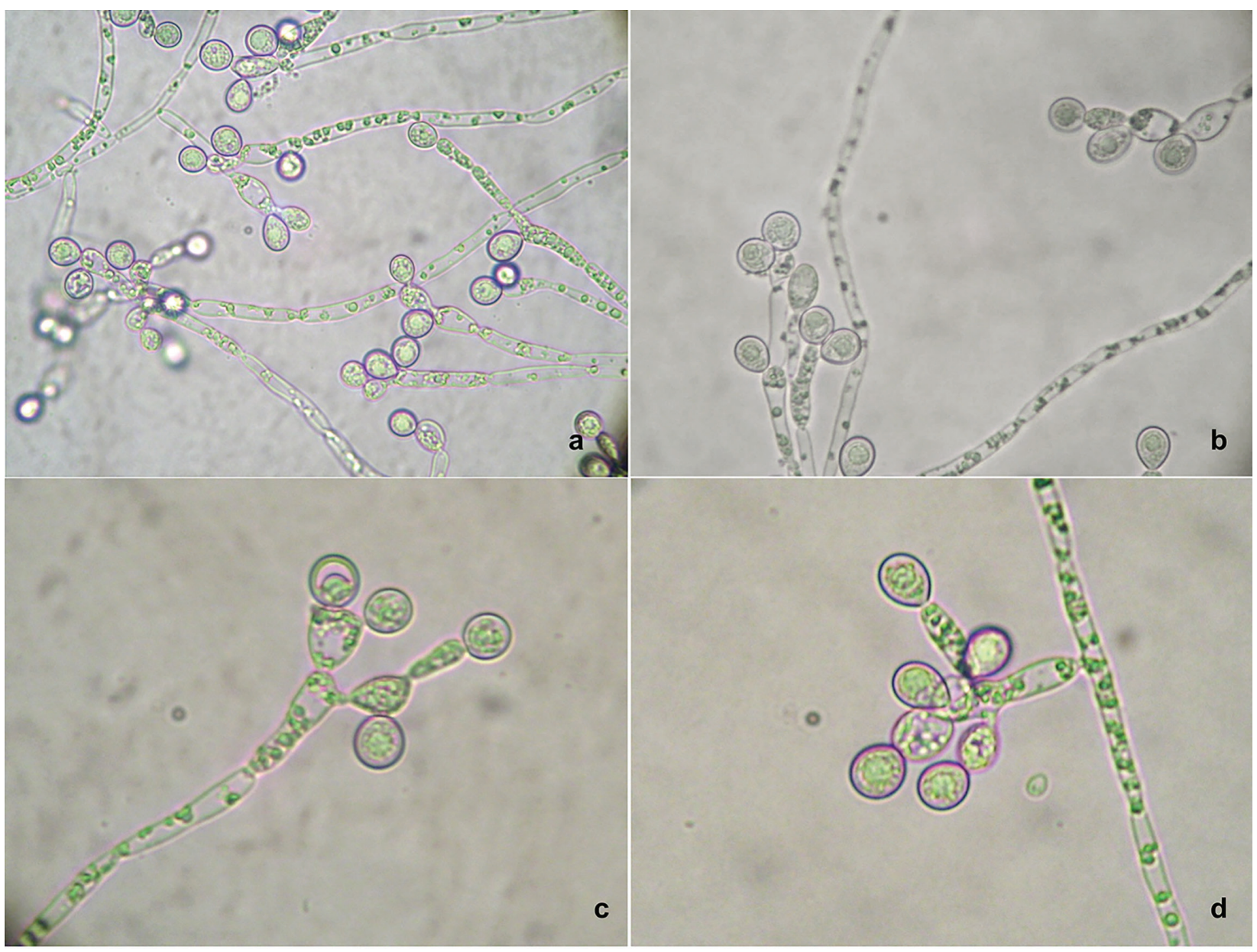

Figure 1 - Micromorphological characteristics: a) Presence of pseudomycelium with 1-5 catenate chlamydospores, mostly in the terminal part (Corn Meal Agar); b), c) and d) Presence of pseudomycelium with terminal chlamydospores, grape-like arranged (STAIB Agar).

habit was not an inclusion criterion in this study. The most important isolates were C. albicans $(81.6 \%)$, followed by C. dubliniensis (5.4\%), and C. parapsilosis (4.1\%).

C. glabrata was the second most frequent species of Candida in the oral mucosa of our ED patients. Candida glabrata emerged as an important opportunistic pathogen in the oral mucosa probably due to its virulence characteristics such as keratinocyte-adherence capacity, extracellular hydrolase production and biofilm-forming ability, besides its innate and acquired resistance to antifungal drugs and its adaptive capacity to different niches in the human body ${ }^{17,20}$. We observed that $C$. glabrata was also found in association with other Candida species (C. albicans, C. parapsilosis and $C$. dubliniensis). Tati et al. ${ }^{21}$ showed that $C$. glabrata coinfection is favored by a pre-established infection with C. albicans, and both species are frequently coisolated in oropharyngeal candidiasis.

C. parapsilosis, the third most isolated species of Candida in this group, is a normal human commensal, frequently isolated from the subungual space of human hands. The habit of provoking vomits by manipulation of the oral cavity would be the potential mode of acquisition of this yeast ${ }^{16}$. We observed that $C$. parapsilosis was found in association with C. albicans. Seabra et al. ${ }^{22}$ reported that both strains, when isolated from cocultures on artificial saliva showed higher adherence and virulence traits in vitro than strains from monocultures.

In two patients $C$. dubliniensis was identified. One was colonized only by $C$. dubliniensis, while the other had blastoconidia and pseudohyphae on DME from different sites (tongue, supragingival area and oral mucosa) that were subsequently identified as $C$. dubliniensis and C. glabrata. The infection in the latter patient was probably related to an impaired immunity secondary to the patient's nutritional deficiency (low body mass index: less than $14.9 \mathrm{Kg} / \mathrm{m}$ ) ${ }^{5,15,18}$.

The frequency of isolation of $C$. dubliniensis is increased in immunocompromised patients: it is found in the oropharyngeal mucosa of HIV-positive/AIDS patients due to its higher capacity of adherence to oral mucosa cells in this group of patients ${ }^{18}$. This species is an infrequent 
component of the normal human flora, being found mainly in the oral cavity, although there are reports of isolation from others anatomical sites ${ }^{23}$. However, recent studies reported an increasing number of $C$. dubliniensis isolated mainly from sputum and alveolar lavage of HIV-negative patients, suggesting that it would have its normal habitat in the upper respiratory tract ${ }^{23}$. In our study, none of the ED patients had severe immunological comorbidities that could predispose to infections by Candida species, thus suggesting that the risk factor for their increased and atypical oral colonization would be the purgative behavior.

The identification of pathogens isolated from clinical specimens generally involves conventional methods based on colony morphologies and biochemical tests that, unfortunately, cannot identify precisely all organisms; therefore, molecular diagnostic methods may be required. The phenotypic characteristics of the isolates (e.g. thermotolerance and chromogenic activity) that lead to the suggestion of $C$. dubliniensis instead of $C$. albicans are debated in the literature ${ }^{8-13,18,24}$. The present study suggests that conventional methods are not sufficient to discriminate C. albicans from C. dubliniensis, and this is an important concern in clinical practice.

The MALDI-TOF MS technique for bacteria and fungi identification up to the genus and species level has been causing a makeover to the workflows for the identification of many pathogens. This technique emerges as a fast, accurate and cost-effective option to replace and/or to complement the conventional phenotypic identification ${ }^{22}$. Additionally, the ability of MALDI-TOF MS to detect a large array of proteins allows the discrimination of closely related species of organisms ${ }^{25}$. However, while MALDI-TOF is not yet widely available to routine mycology laboratories in Latin America, the low cost, classical mycology techniques are accessible to these laboratories and they still hold an important place in the diagnosis of medically important fungi.

In conclusion, we suggest that alterations of the natural microbiota due to purgative habits might predispose to the acquisition of non-albicans species, such as C. glabrata (a species frequently isolated in association with $C$. albicans in oropharyngeal candidiasis), C. parapsilosis (a species frequently isolated from the subungual space of human hands) and C. dubliniensis (part of the normal flora of the upper respiratory tract). Unlike $C$. albicans, these species may eventually lead to oral candidiasis in these patients. Further studies on the prevalence and severity of oral candidiasis in ED patients are warranted.

\section{ACKNOWLEDGMENTS}

DYT conducted this work supported by a Fapesp postdoctoral fellowship (2018/15491); GB conducted this work with finacial support from Fapesp, grant $\mathrm{N}^{\circ}$ 2016/08730-6, and Conselho Nacional de Desenvolvimento Científico e Tecnológico. We are grateful to the anonymous reviewers for their many suggestions, which helped improving the manuscript.

\section{CONFLICT OF INTERESTS}

We have no conflict of interests or disclosures to declare.

\section{REFERENCES}

1. Klump KL, Bulik CM, Kaye WH, Treasure J, Tyson E. Academy for eating disorders position paper: eating disorders are serious mental illnesses. Int J Eat Disord. 2009;42:97-103.

2. Uhlen MM, Tveit AB, Stenhagen KR, Mulic A. Self-induced vomiting and dental erosion: a clinical study. BMC Oral Health. 2014;14:92.

3. Dynesen AW, Bardow A, Petersson B, Nielsen LR, Nauntofte B. Salivary changes and dental erosion in bulimia nervosa. Oral Surg Oral Med Oral Pathol Oral Radiol Endod. 2008;106:696707.

4. Lo Russo L, Campisi G, Di Fede O, Di Liberto C, Panzarella V, Lo Muzio L. Oral manifestations of eating disorders: a critical review. Oral Dis. 2008;14:479-84.

5. Chanda W, Joseph TP, Wang W, Padhiar AA, Zhong M. The potential management of oral candidiasis using anti-biofilm therapies. Med Hypotheses. 2017;106:15-8.

6. Akgül Ö, Çerikçioğlu N. Hypertonic sabouraud dextrose agar as a substrate for differentiation of Candida dubliniensis. Mycopathologia. 2009;167:357-9.

7. Coleman DC, Rinaldi MG, Haynes KA, Rex JH, Summerbell RC, Anaissie EJ, et al. Importance of Candida species other than Candida albicans as opportunistic pathogens. Med Mycol. 1998;36 Suppl 1:156-65.

8. Matic Petrovic S, Radunovic M, Barac M, Kuzmanovic Pficer J, Pavlica D, Arsic Arsenijevic V, et al. Subgingival areas as potential reservoirs of different Candida spp in type 2 diabetes patients and healthy subjects. PLoS One. 2019;14:e0210527.

9. Vieira Esteves C, Gushiken de Campos W, Gallo RT, Ebling Artes G, Shimabukuro N, Witzel AL, et al. Oral profile of eating disorders patients: case series. Spec Care Dentist. 2019;39:572-7.

10. de Hoog GS, Guarro J, Gené J, Figueras MJ. Atlas of clinical fungi. $2^{\text {nd }}$ ed. Utrecht: Centraalbureau voor Schimmelcultures; 2000.

11. Gales AC, Pfaller MA, Houston AK, Joly S, Sullivan DJ, Coleman DC, et al. Identification of Candida dubliniensis based on temperature and utilization of xylose and $\alpha$-methylD-glucoside as determined with the API 20C AUX and Vitek YBC systems. J Clin Microbiol. 1999;37:3804-8. 
12. Pinjon E, Sullivan D, Salkin I, Shanley D, Coleman D. Simple, inexpensive, reliable method for differentiation of Candida dubliniensis from Candida albicans. J Clin Microbiol. 1998;36:2093-5.

13. Chowdhary A, Randhawa HS, Kowshik T, Kathuria S, Roy P, Brandt ME. Application of hypertonic Sabouraud glucose agar for differentiation of Candida dubliniensis from Candida albicans. Diagn Microbiol Infect Dis. 2011;69:440-2.

14. Morgan XC, Segata N, Huttenhower C. Biodiversity and functional genomics in the human microbiome. Trends Genet. 2013;29:51-8.

15. Rossoni RD, Barbosa JO, Vilela SF, Jorge AO, Junqueira JC. Comparison of the hemolytic activity between C. albicans and non-albicans Candida species. Braz Oral Res. 2013;27:484-9.

16. Trofa D, Gácser A, Nosanchuk JD. Candida parapsilosis: an emerging fungal pathogen. Clin Microbiol Rev. 2008;21:606 25.

17. Rodrigues CF, Rodrigues ME, Silva S, Henriques M. Candida glabrata biofilms: how far have we come? J Fungi (Basel). 2017;3:E11.

18. Livério HO, Ruiz LS, Freitas RS, Nishikaku A, Souza AC, Paula $\mathrm{CR}$, et al. Phenotypic and genotypic detection of candida albicans and candida dubliniensis strains isolated from oral mucosa of AIDS pediatric patients. Rev Inst Med Trop Sao Paulo. 2017;59:e14.
19. Back-Brito GN, Mota AJ, Souza Bernardes LÂ, Takamune SS, Prado EF, Cordás TA, et al. Effects of eating disorders on oral fungal diversity. Oral Surg Oral Med Oral Pathol Oral Radiol. 2012;113:512-7.

20. Li L, Redding S, Dongari-Bagtzoglou A. Candida glabrata: an emerging oral opportunistic pathogen. J Dental Res. 2007;86:204-15.

21. Tati S, Davidow P, McCall A, Hwang-Wong E, Rojas IG, Cormack $\mathrm{B}$, et al. Candida glabrata binding to Candida albicans hiphae enables its development in oropharyngeal candidiasis. PLoS Pathog. 2016;12:e1005522.

22. Seabra CL, Botelho CM, Henriques M, Oliveira R. Differential adherence and expression of virulence traits by Candida albicans and Candida parapsilosis in mono- and dual-species cultures in artificial saliva. Mycopathologia. 2013;176:33-40.

23. Fotedar R, Al Hedaithy SS. Prevalence of Candida dubliniensis among germ tube-positive yeasts recovered from the respiratory specimens in HIV-negative patients. Mycoses. 2004;47:150-5.

24. de Loreto ES, Pozzatti P, Alves Scheid L, Santurio D, Santurio JM, Alves SH. Differentiation of Candida dubliniensis from Candida albicans on rosemary extract agar and oregano extract agar. J Clin Lab Anal. 2008;22:172-7.

25. Chalupová J, Raus M, Sedlářová M, Šebela M. Identification of fungal microorganisms by MALDI-TOF mass spectrometry. Biotechnol Adv. 2014;32:230-41. 